\title{
Etiology of vaginal discharge in sexually inactive adolescents
}

\author{
Yasemin Düzçeker ${ }^{1}$, Sinem Akgül ${ }^{1}$, Yasemin Özsürekci ${ }^{2}$, Orhan Derman ${ }^{1}$, Ateş Kara ${ }^{2}$, \\ Nuray Kanbur \\ Divisions of ${ }^{1}$ Adolescent Medicine and 2Pediatric Infectious Diseases, Department of Pediatrics, Hacettepe University Faculty \\ of Medicine, Ankara, Turkey. E-mail: nuraykanbur@hotmail.com \\ Received: 30th March 2017, Accepted: 13th September 2018
}

Vaginal discharge (VD) is common during puberty and may be physiological however it might also be the result of infection and other conditions. ${ }^{1}$ The aim of this study was to determine the causes for VD in sexually inactive adolescents and to evaluate clinical and laboratory data which may assist in determining the etiology for infectious VD.

\section{Material and Methods}

The study took place at Hacettepe University, Department of Pediatrics, Division of Adolescent Medicine between September 2015 and March 2016. All sexually inactive pubertal patients with a complaint of VD were included. Clinical and laboratory data consisted of signs (perineal and vaginal hyperaemia) and symptoms (odor of discharge and perineal itching), vaginal $\mathrm{pH}$, Gram stain and culture. In culture results, colonization was considered according to clinical findings as well as bacterial growth in culture. This retrospective analysis of patient records was approved by the Hacettepe Research Ethics Review Board (B.30.2.ANK.0.20.05.04-8772). Statistical analyses were performed using SPSS version 19.0 (Chicago, IL, USA). Descriptive statistics were used to summarize the participants' baseline characteristics. $\mathrm{P}$ values were calculated using the Chi square. A $p$ value $<0.05$ was considered significant.

\section{Results}

Fifty-six sexually inactive pubertal patients between the ages of 10-18 years (mean age $14.6 \pm 1.6$ years) were included. Culture results revealed normal vaginal flora in 25 (44.6\%), candida in $19(33.9 \% ; 11$ C. albicans, 8 nonalbicans), bacterial vaginosis in $6(10.7 \%$; 3 Gardnerella vaginalis, 1 Streptococcus agalactiae, 1 Klebsiella pneumoniae and 1 Klebsiella oxycota + Escherichia coli) and Gram (-) colonization in $6(10.7 \%)$. Gram staining revealed yeast in
$10(17.9 \%)$ patients but culture results were negative in terms of candida species in 4 (40\%). The distribution of vaginal culture results was significantly different when evaluated according to vaginal $\mathrm{pH}(\mathrm{p}=0.004$; Fig. 1$)$.

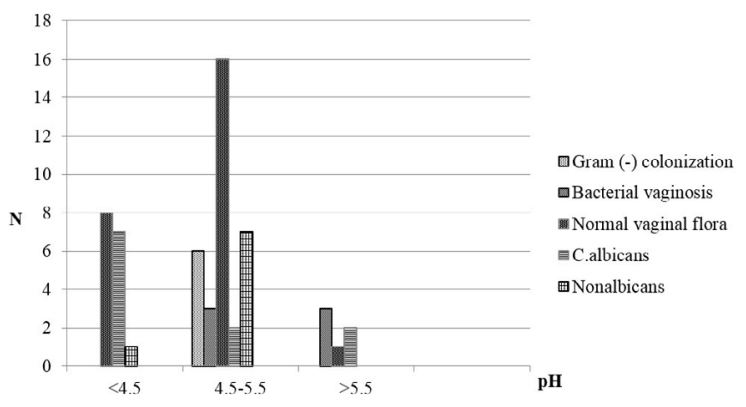

Fig. 1. Vaginal culture results according to $\mathrm{pH}$

\section{Discussion}

In this study, physiologic leucorrhoea was the most common cause of VD in sexually inactive adolescents whereas the most common infectious causes of VD were candida and bacterial vaginosis with lower rates when compared to the literature. ${ }^{2}$ Also, nonalbicans candida species were nearly as common as $C$. albicans. ${ }^{2}$ The novel finding of this study was that infectious agents were more commonly C. albicans when vaginal $\mathrm{pH}$ was $\leq 4.5$ and nonalbicans candida when vaginal $\mathrm{pH}$ was 4.5-5.5 in sexually inactive pubertal girls. Also, bacterial colonization was detected only when the $\mathrm{pH}$ was 4.5-5.5.

In conclusion, we suggest that determination of vaginal $\mathrm{pH}$, due to the fact that it is a non-invasive and inexpensive procedure, is important in predicting etiology of infectious VD in sexually inactive adolescents. 


\section{REFERENCES}

1. Braverman PK, Breech L; The Committee on Adolescence. Gynecologic examination for adolescents in the pediatric office setting. Pediatrics 2010; 126 : 583-590.
2. Freeto JP, Jay MS. What's really going on down there? A practical approach to the adolescent who has gynecologic complaints. Pediatr Clin North Am 2006; 53: 529-545. 